\title{
Prevalence of Impostor Phenomenon in Physiotherapy Professionals: A Pan India Survey
}

\author{
Vaishvi. P. Kansara ${ }^{\text {i }}$ Neeraj Kumar ${ }^{\text {ii }}$, Sukhpreet Pabla ${ }^{\text {iii }}$ \\ ${ }^{\mathrm{i}}$ Intern, ${ }^{\mathrm{ii}}$ Professor and HOD, ${ }^{\mathrm{iii}}$ Assistant Professor, \\ Dr. APJ Abdul Kalam College of Physiotherapy, Pravara Institute of Medical Sciences, \\ Deemed to be University, Loni, Maharashtra, India. \\ Corresponding Author: Vaishvi Kansara
}

\begin{abstract}
Background: Impostor Phenomenon is described as the psychological experience in which there is the individual does not have the ability to believe that the accomplishments achieved by him/her are deserved or are achieved because of one's own skills and talents, but because of one being fortunate. Impostor Phenomenon is seen in many professional setting and is also prominent in highly successful individuals. Impostor phenomenon is increasing among the health care professionals. The following study determines the prevalence of Impostor Phenomenon in Physiotherapy professionals.

Method: The study was conducted through online survey method. Simple random sampling was done and forms were sent to the physiotherapists. 200 participants responded to the questionnaire. Clance Impostor Phenomenon Scale was used to measure Impostor Phenomenon. Demographic data was collected and questionnaire was filled by the participants. Total scoring was done and the participants were classified according to categories of Clance Impostor Phenomenon Scale.

Result: $7.5 \%$ of physiotherapists had few impostor characteristics, $58 \%$ experienced moderate impostor characteristics, $32 \%$ having often impostor characteristics and $2.5 \%$ of had intense Impostor Phenomenon. The scoring of male and female physiotherapists was similar and the scores of clinicians were more than academicians.

Conclusion: The study concluded that Impostor Phenomenon affected the Physiotherapists. Many of physiotherapists scored between 40-60 and indicated having the characteristics of Impostor Phenomenon at a moderate level. No significant difference was seen between males and females. The prevalence of Impostor Phenomenon in clinicians was higher than in academicians.
\end{abstract}

Keywords: Impostor Phenomenon, Health care professionals, Physiotherapists.

\section{INTRODUCTION}

Impostor Phenomenon is described as the psychological experience in which there is the individual does not have the ability to believe that the accomplishments achieved by him/her are deserved or are achieved because of one's own skills and talents, but because of one being fortunate. ${ }^{1}$ Clance first identified this phenomenon in high achieving professional women, ${ }^{2}$ but recent research finds the prevalence of this phenomenon is seen in males too. ${ }^{3}$ The person may feel doubt, shame or fear of being a fraud and also lack of self- confidence. This psychological characteristic acts as a disturbance into one's success. It deprives the person from moving forward towards success. Using their hidden talents impostors do not look up for their achievements and deny them. ${ }^{4}$

\section{Mental health and Impostor Phenomenon:}

Impostor Phenomenon affects the mental health of the individual. Psychological distress, mental health disorders such as anxiety, depression, low self-esteem and social dysfunctions can be 
caused by Impostor Phenomenon. 5, 6 Positive correlations were found with depression, anxiety and self-consciousness which are the three of the six traits of Neuroticism facet and negative correlations were found with Conscientiousness facet of the Revised NEO Personality Inventory. ${ }^{7,8}$

\section{Perfectionism and Impostor Phenomenon:}

There is a positive co-relation between Perfectionism and Impostor Phenomenon. People with Impostor Phenomenon are not satisfied for what they have achieved. They expect that whatever task they perform must give perfect results and hence they overwork to meet perfectionism. ${ }^{9}$ Impostor Phenomenon can also occur if the individual has numerous roles and there is imbalance between the management of the roles in a perfect manner. $^{10}$

\section{Self efficacy and Impostor Phenomenon:}

Impostor Phenomenon are
negatively co-related with each other .Individuals with a low self efficacy will question their abilities and will have a dread of getting evaluated by others and then eventually fear of failure in front of their peers leading to Impostor Phenomenon. ${ }^{11,12}$

Clance and Imes (1978) identified the behaviors that maintain the Impostor Phenomenon and discovered a pattern of behaviours which reinforced the Impostor Phenomenon. The cycle begins when the person is given a new opportunity or some new challenging task. The person will either over prepare thinking that he/she should not lag from others or will procrastinate the preparation and then do it in a hurry. If the outcome of the task or the opportunity is successful then the person starts thinking that the success is due to the hard work or because he/she was lucky enough to gain the success. The person then gains the external recognition of the performance. After success the person starts developing the feeling of dread, worry and doubt of the gained success. Success and positive response from the environment build up the whole behavior pattern and cycle gets repeated. ${ }^{2,13}$ Solving a task which does not take any efforts makes them feel that it was very easy and anybody in the world can do it. But however if the task is difficult and they successfully do it, the credits are given to their fate and hard work and not to their intelligence and smartness. ${ }^{9}$ Dr. Pauline Clance once said in an interview that in his therapy practice he had seen many lawyers, doctors and business executives who experienced Impostor Phenomenon. ${ }^{11}$

Students taking admission in medical school come across unique stresses, challenges, social expectations. At social gatherings they are being asked about the diagnosis and treatment of their illness even if the student has just entered the medical school or in the half way of it. Also their peers are smart and intelligent which make them think incompetent 14 and hence they develop the characteristics of Impostor Phenomenon.

Becoming a health care professional demands compassion, passion, strong work ethic, confidence, professionalism and higher self-efficacy. It is a profession which involves proper decision making quality and experience. ${ }^{15}$ If the clinician is confident, the ability of the clinician to assess and manage the patient increases. This, in turn, enhances the patient's quality of life. From a medical student to a health care professional the development of Impostor Phenomenon can occur at any stage of the career. $^{14}$ Health care professionals who think that they are inadequate and have self-doubt will not be able to treat the patients with the required potential. ${ }^{15}$ Self efficacy would play a major role in a person with a health care profession. Hence as mentioned above about the relation between self efficacy and Impostor Phenomenon, professionals would start losing their quality of decision making which would have a negative impact on their professional growth. ${ }^{4}$ The prevalence of Impostor Phenomenon is increasing among the health care professionals. ${ }^{4,16}$ 
Physiotherapy is a health care profession that assists people in restoring, maintaining, and optimizing their strength, work, movement, and general well-being. It involves proper assessment, evaluation, diagnosing, and treating a range disabilities and disorders. In order to manage patients, Physiotherapists must inculcate skills and qualities which enhance the quality of life of the patients. ${ }^{17}$ Often therapists doubt about their skills and abilities and this feeling of inadequacy and self doubt leads to Impostor Phenomenon. The professional growth and skill development of the therapist gets hampered which leads to inability to manage the cases with reasonable logic and expertise. $^{15}$

Physiotherapists with low selfefficacy may have feeling of 'self-doubt' that whether the application of a particular therapy is appropriate for that particular patient. This feeling of self-doubt then leads to Impostor Phenomenon. There is a lack of evidence on Physiotherapists experiencing this particular phenomenon. Thus the following study is conducted to determine the impact of Impostor Phenomenon on Physiotherapy professionals.

\section{MATERIAL AND METHOD}

The present study is a cross sectional study which was conducted through online survey method. The study population of the study was Physiotherapy professionals across India and the sample size was 200 Physiotherapists. Simple random sampling was used. Clance Impostor Phenomenon Scale was used as the measure for Impostor Phenomenon. It is 20 item scale with 5 Likert points 5 (strongly agree) to 1 (strongly disagree). Ethical approval was done by the ethics committee. After the approval, the questionnaire was circulated to the Physiotherapists through social media platforms. First section included Informed consent which was taken from the participants. After the informed consent was taken, the second section included demographic data which consisted of age, gender, city, state, qualification, designation and years of experience. The Physiotherapists were then selected on the basis of eligibility criteria which included the participants willing to participate, Physiotherapists practicing in India and Physiotherapists who have at least completed either graduation. Participants having other mental problems not related to Impostor Phenomenon were excluded. The third section of the form consisted of The Clance Impostor Phenomenon Scale which was filled by the participants. The data analysis was done on the basis of the questionnaire filled by the Physiotherapists. The answers and results were noted.

\section{RESULT}

The aim and objective of the study was to determine the prevalence of Impostor Phenomenon in male and female physiotherapy professionals, in academicians and clinicians and in beginners and experienced physiotherapists. The results were analyzed on the basis of the Google forms filled by the participants. The participants were selected on the basis of the eligibility criteria. The study received 205 responses from all over India out of which 200 participants were selected according to the eligibility criteria. Demographic details were collected. After the demographic details the questionnaire consisted of 20 item scale with 5 likert points: 5 (strongly agree) to 1 (strongly disagree). According to the responses for each question, total score was calculated with the maximum possible score of 100 points. The participants were then categorized into 4 categories according to their score. The categories according to CIPS were: few IP characteristics (score: 040), moderate IP characteristics (score: 4160 ), often IP characteristics (score: 61-80), and intense IP characteristics (score: 81100).

\section{1) Demographics:}

From the selected participants 132 $(66 \%)$ were females and $68(32 \%)$ were males. The mean age difference in males was $26.36 \pm 4.73$ years and in females was 
Vaishvi. P. Kansara et.al. Prevalence of impostor phenomenon in physiotherapy professionals: a pan India survey.

$25.00 \pm 3.94$ years. $189(95 \%)$ participants were clinicians and $11(5 \%)$ participants were academicians. To find the prevalence of Impostor Phenomenon in beginners and experienced professionals years of experience was taken.
Table 1. Mean and standard deviation of age of males and female

\begin{tabular}{|c|c|}
\hline Gender & Mean \pm SD \\
\hline Male & $26.36 \pm 4.73$ \\
\hline Female & $25.00 \pm 3.94$ \\
\hline
\end{tabular}

Number of Male and Female Physiotherapists

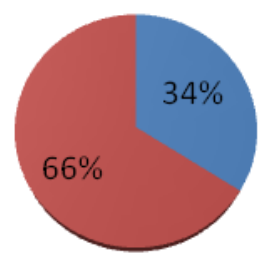

males

females

Fig.1: Number of Male and Female responded

\section{Number of Academician and Clinician}

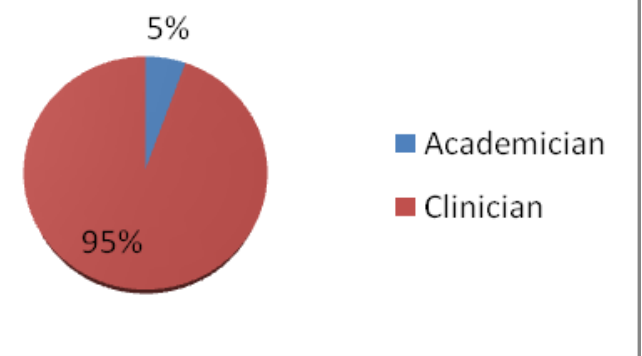

Fig.2: Number of Academician and Clinician responded

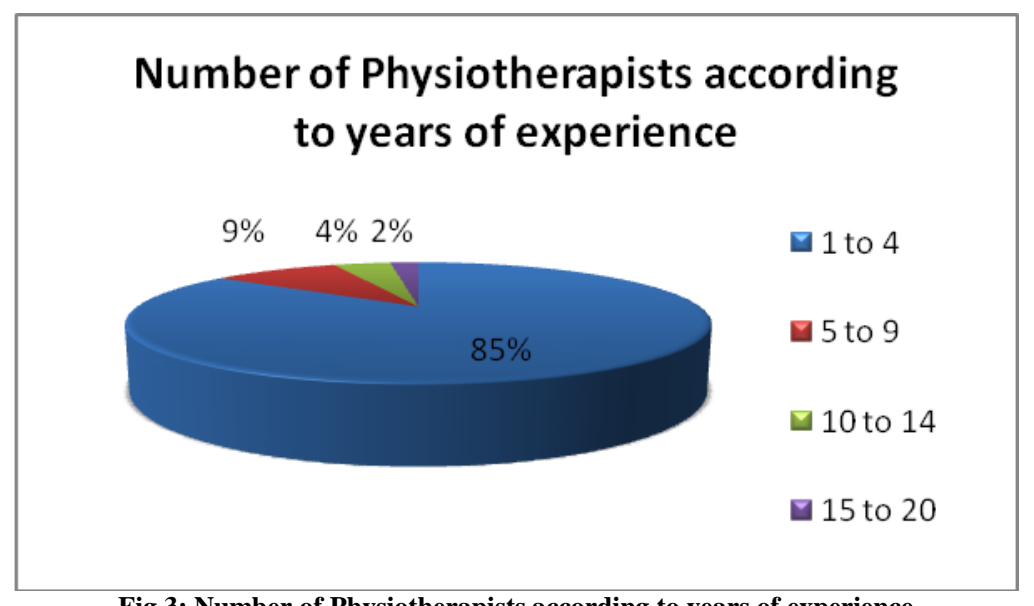

Fig.3: Number of Physiotherapists according to years of experience

\section{2) Categorization Of Physiotherapists According To CIPS:}

Total scoring was done and the percentage of the Physiotherapists in each category was analyzed.

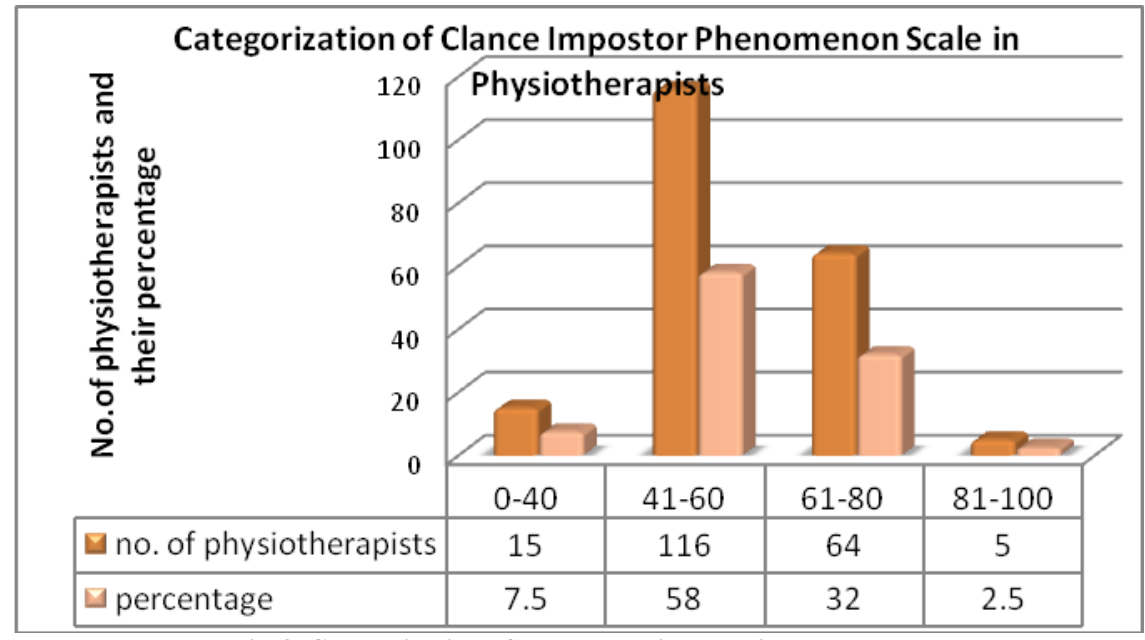

Fig.4: Categorization of all the Physiotherapists responded 
Vaishvi. P. Kansara et.al. Prevalence of impostor phenomenon in physiotherapy professionals: a pan India survey.

\section{3) Categorization of Males and Females:}

Females responded more than males. Out of 68 males and 132 females responded and were then categorized and the percentage was calculated.

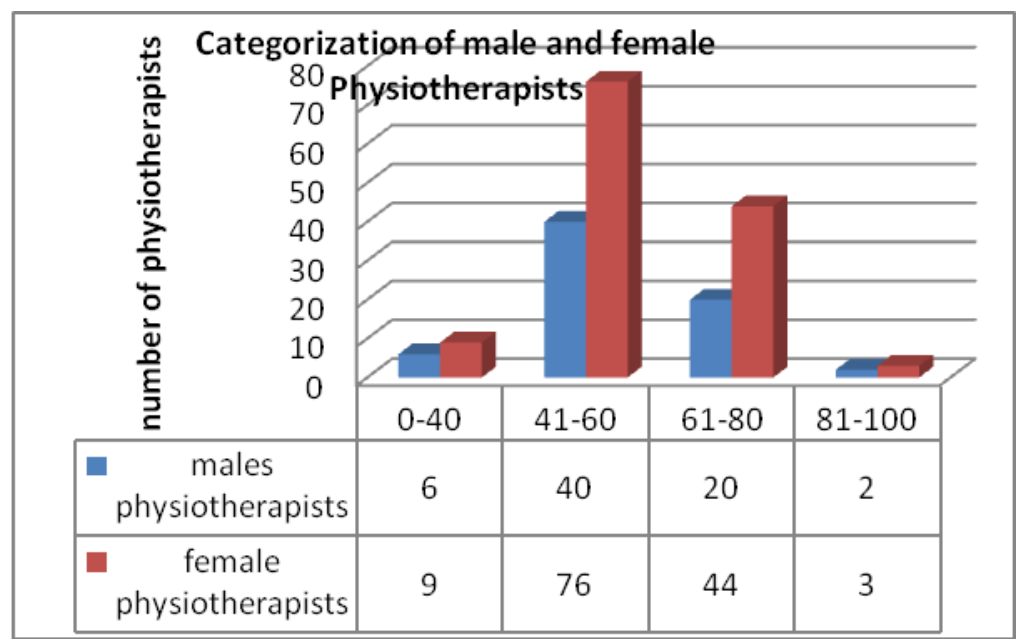

Fig.5: Categorization of Male and Female Physiotherapists

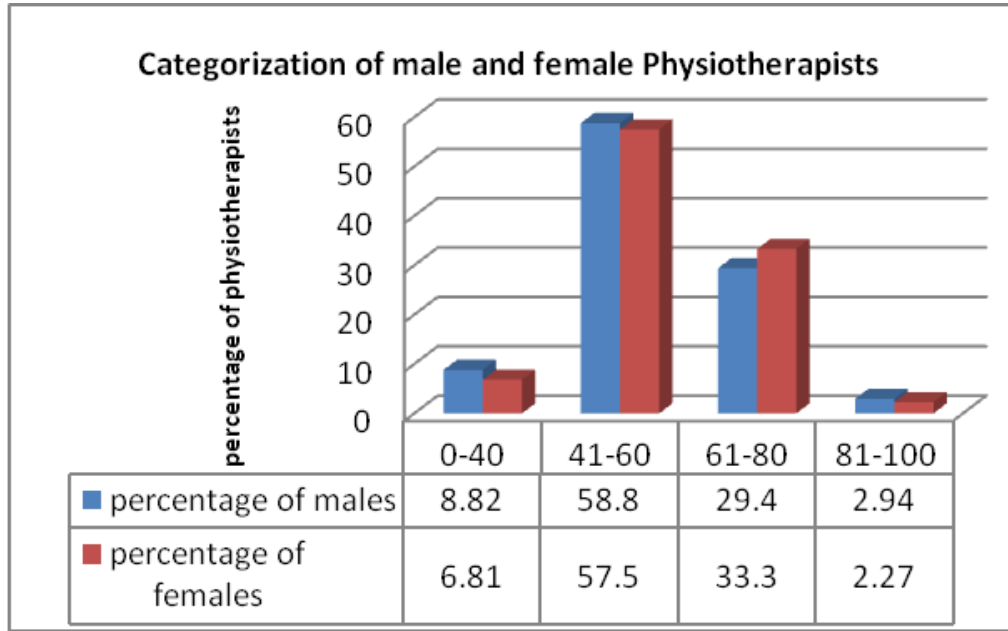

Fig.6: Categorization of Male and Female Physiotherapists in percentage

\section{4) Categorization Of Clinicians And Academicians:}

95\% of clinicians and 5\% academicians responded to the survey. The therapists were then classified according to CPIS scale and percentage was calculated.

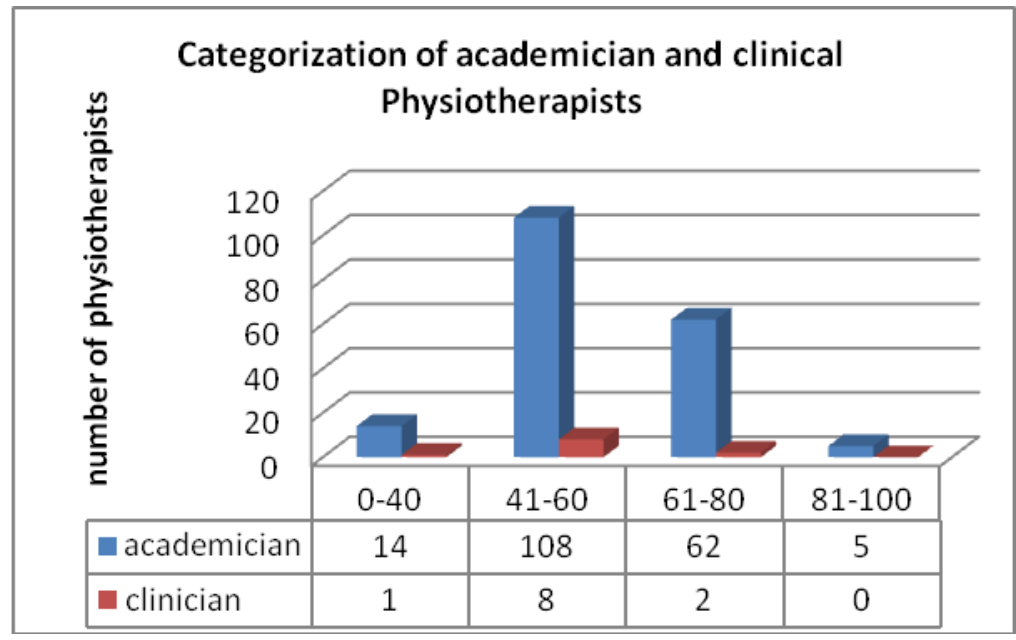

Fig.7: Categorization of Academician and Clinicians 
Vaishvi. P. Kansara et.al. Prevalence of impostor phenomenon in physiotherapy professionals: a pan India survey.

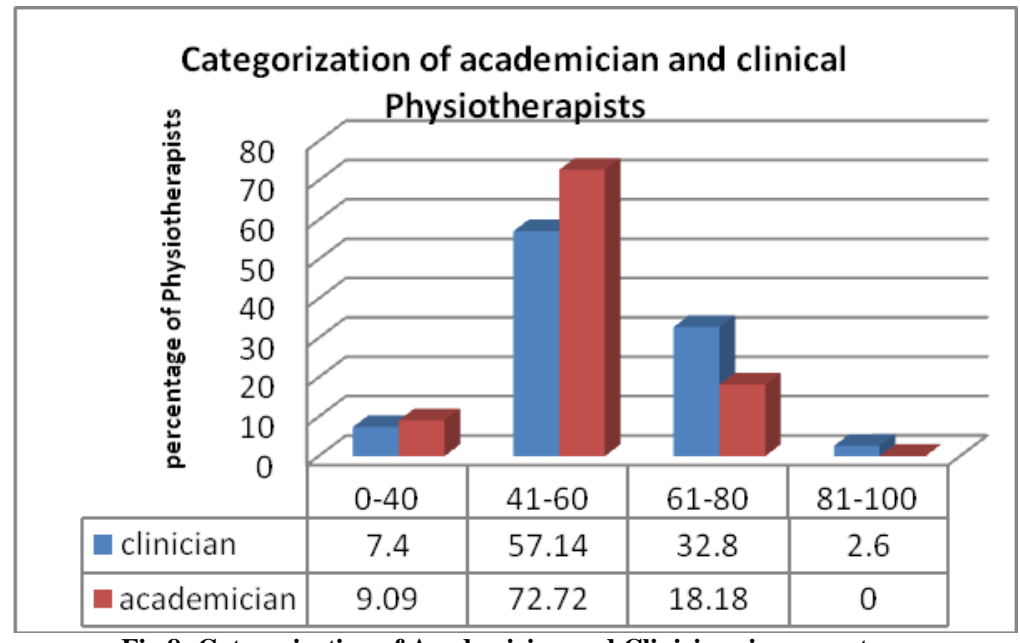

Fig.8: Categorization of Academician and Clinicians in percentage

\section{5) Categorization according to years of experience:}

Participants were classified on the basis of their years of experience and then were categorized according to CIPS scale.

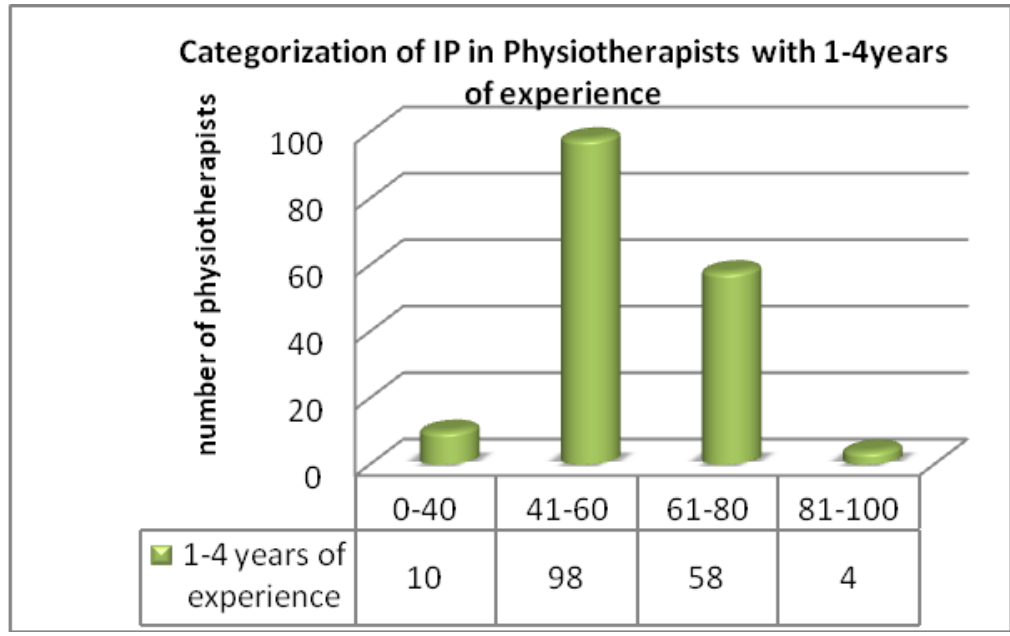

Fig.9: Categorization with 1-4 years of experience

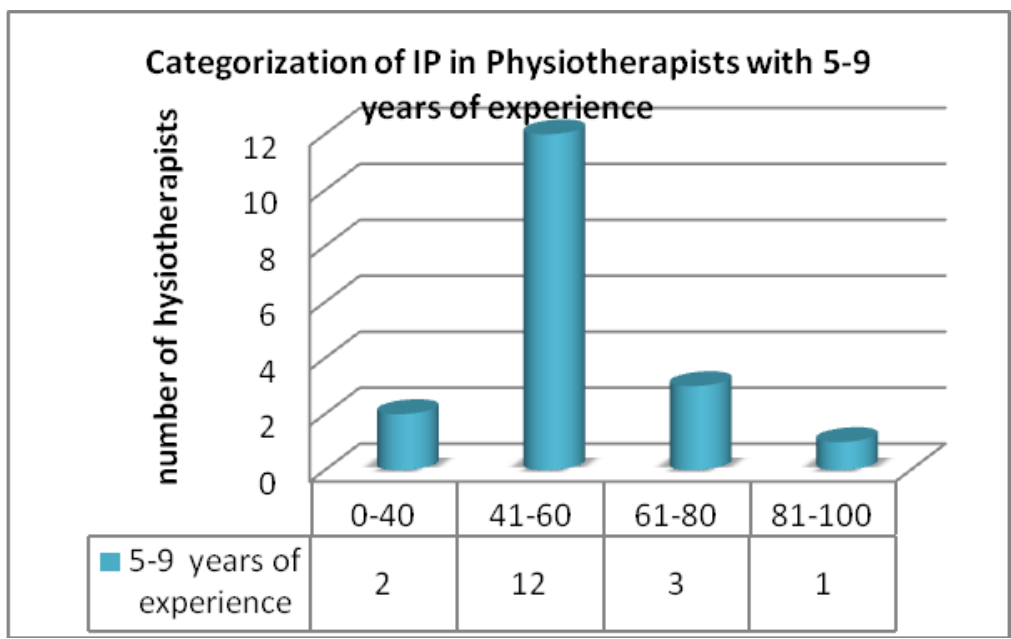

Fig.10: Categorization with 5-9 years of experience 
Vaishvi. P. Kansara et.al. Prevalence of impostor phenomenon in physiotherapy professionals: a pan India survey.

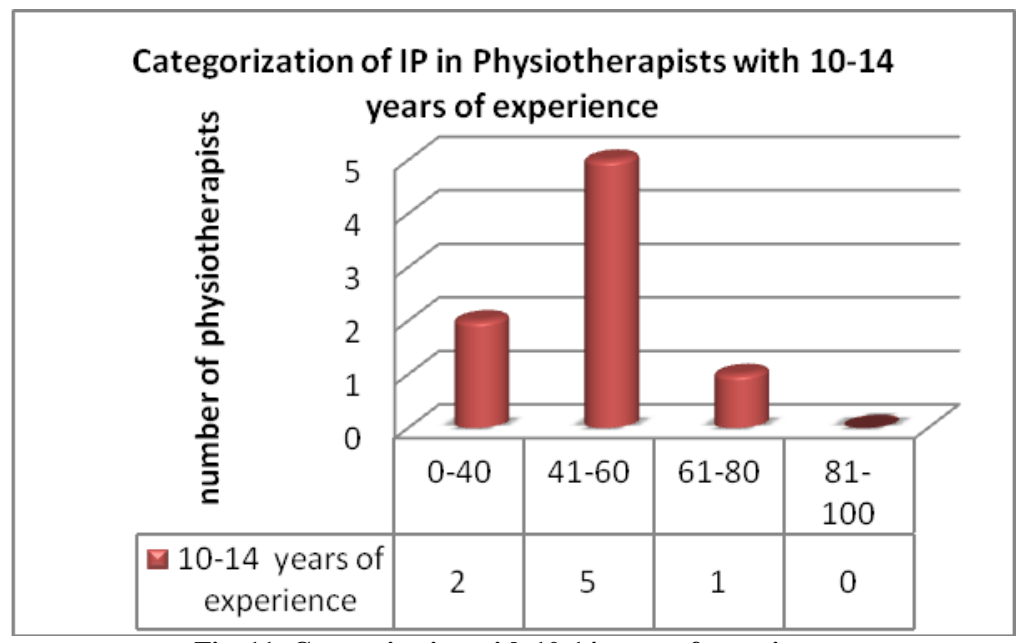

Fig..11: Categorization with 10-14 years of experience

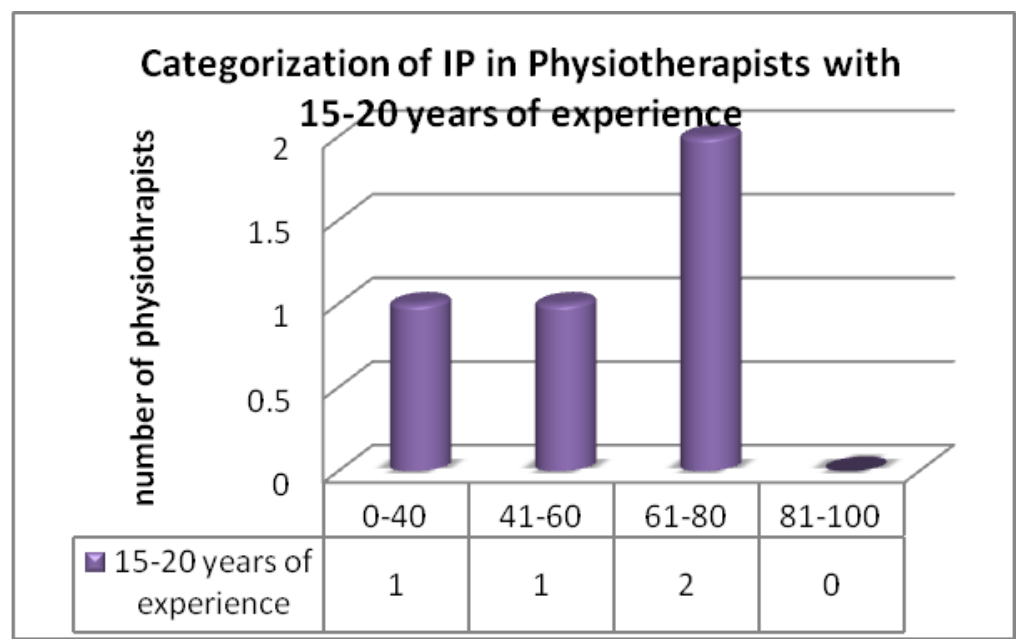

Fig.12: Categorization with 15-20 years of experience

\section{DISCUSSION}

As Physiotherapy is a growing field in India. ${ }^{18}$ The demand of an appropriate knowledge and accuracy to clinical decision making is high. ${ }^{19}$ This sometimes leads to self doubt immerging a feeling of impostor. This study is carried out to find how this feeling takes place in physiotherapy professionals.

Online survey was conducted via Google forms and responses were taken from the Physiotherapists all over India. A questionnaire was sent consisting of 20 item scale with 5 Likert points: 5 (strongly agree) to 1 (strongly disagree). 200 participants were selected according to the inclusion criteria. Responses were obtained from various states of India among which highest responses were obtained from Maharashtra state. According to the responses for each question, total score was calculated with the maximum possible score of 100 points.

The study proceeded with selected participants, where $132(66 \%)$ were females and $68(32 \%)$ were males. $189(95 \%)$ participants were clinicians and 11 (5\%) participants were academicians. To find the prevalence of Impostor Phenomenon in beginners and experienced professionals years of experience was included and the responses were divided among categories according to the Clance Impostor Phenomenon Scale and studied accordingly. Here initial category was among the male and female responses. Out of 68 males, 6 $(8.82 \%)$ males were found to have few impostor characteristics, $40(58.8 \%)$ were found to have moderate impostor characteristics, 20 (29.4\%) had often impostor characteristics and only 2 (2.94\%) males had intense impostor characteristics. 
A research by Topping (1983) 'The Imposter Phenomenon in Higher Education: Incidence and Impact' found that men had a slightly higher mean Impostor Phenomenon score than women in a survey of male and female faculty members ${ }^{20}$ Among 132 female Physiotherapists who responded, 9 $(6.81 \%)$ were categorized having few impostor characteristics, $76(57.5 \%)$ females had moderate impostor characteristics, 44 (33.3\%) females were found to have often impostor characteristics and only $3(2.27 \%)$ females had intense impostor characteristics. Similar results were seen in the previous studies indicating no significant difference in males and females. $21,22,23,24,25$ In the study titled 'Family Medicine Residents and the Impostor Phenomenon' conducted by Kathy Oriel (et al.), females were more likely than males to be identified as impostors. ${ }^{26}$ There are similar also studies which shows that the prevalence of Impostor Phenomenon in seen more in females. ${ }^{2,6,21,27}$

As the study progressed the category took in consideration was of academician and clinician, where $95 \%$ of clinicians and $5 \%$ academicians showed their interest in study. From 189 clinicians, 14 had few impostor characteristics, 108 Physiotherapists had moderate impostor characteristics, 62 were categorized as often impostor characteristics and 5 experienced intense impostor characteristics. Only 1 of the 11 academicians experienced few impostor characteristics, 8 had moderate impostor characteristics 2 had often characteristics and none of them had intense Impostor Phenomenon. Hence we found out that the prevalence of Impostor Phenomenon is more in clinicians than the academicians, as they need to take more practical and confident actions related to patient diagnosis and treatment. In a study conducted on IP in hospitalists it was concluded that 24 out of 71 hospitalists that is $33 \%$ scored above 60 and were considered to have IP. ${ }^{28} 35 \%$ of clinicians in our study have scored above 60 which is similar to the hospitalists study.
In the initial years of physiotherapy experience, guidance is provided at its best. A shift of character occurs as the year of experience increases, leading to an increased expectance of correct diagnosis and treatment causing a spike in impostor characteristics among this population. Also projected in the study, here participants with more experience in the field show increased impostor characteristics. With an experience of 15-20 years in the field of physiotherapy, among the responders in this category maximum scored between 61 to 80 indicating often impostor characteristics. Physiotherapists with a minimum year of experience of 1 to 4 years, Scored in between 41-60 representing moderate impostor phenomenon.

Depending on the total responses of 200 Physiotherapists all over India and their representing score of CIPS, It shows that $7.5 \%$ of Physiotherapists had few impostor characteristics, $58 \%$ experienced moderate, $32 \%$ having quite often and only $2.5 \%$ of had intense Impostor Phenomenon. Some similar studies were done in different geographical areas and professions with similar results. $3,4,6,15,16,21,26,22,27,29,30$

\section{CONCLUSION}

The study concluded that Impostor Phenomenon affected the Physiotherapists but the prevalence of intense Impostor phenomenon was very less. Many of physiotherapists scored between 40-60 which means that they have the characteristics of IP but at a moderate level. Also the study concluded that gender does not play a significant role in this phenomenon because no difference was found among both the genders. According to the study prevalence of Impostor Phenomenon in clinicians was higher than in academicians.

\section{Acknowledgement: None}

Conflict of Interest: None

Source of Funding: None 


\section{Ethical Approval: Approved}

\section{REFERENCES}

1. Langford J, Clance PR. The imposter phenomenon: recent research findings regarding dynamics, personality and family patterns and their implications for treatment. Psychotherapy: theory, research, practice, training. 1993;30(3):495.

2. Clance PR, Imes SA. The imposter phenomenon in high achieving women: Dynamics and therapeutic intervention. Psychotherapy: Theory, Research \& Practice. 1978;15(3):241.

3. Parkman A. The imposter phenomenon in higher education: Incidence and impact. Journal of Higher Education Theory and Practice. 2016 Feb 1;16(1):51.

4. Kamarzarrin H, Khaledian M, Shooshtari M, Yousefi E, Ahrami R. A study of the relationship between self-esteem and the imposter phenomenon in the physicians of Rasht city. European Journal of Experimental Biology. 2013;3(2):363-6.

5. Chrousos GP, Mentis AF. Imposter syndrome threatens diversity. Science. 2020 Feb 14;367(6479):749-50.

6. Bravata DM, Watts SA, Keefer AL, Madhusudhan DK, Taylor KT, Clark DM, Nelson RS, Cokley KO, Hagg HK. Prevalence, predictors, and treatment of impostor syndrome: a systematic review. Journal of General Internal Medicine. 2020 Apr;35(4):1252-75.

7. Kolligian $\mathrm{Jr} \mathrm{J}$, Sternberg RJ. Perceived Fraudulence in Young Adults: Is There an 'Imposter Syndrome'? Journal of personality assessment. 1991 Apr 1;56(2): 308-26.

8. Chae JH, Piedmont RL, Estadt BK, Wicks RJ. Personological evaluation of Clance's Imposter Phenomenon Scale in a Korean sample. Journal of personality assessment. 1995 Dec 1;65(3):468-85.

9. Filarowska M, Schier K. The impostor phenomenon-the sense of intellectual falseness. Psychoterapia. 2018 Jan 1(2):3545.

10. Cusack CE, Hughes JL, Nuhu N. Connecting gender and mental health to imposter phenomenon feelings. Psi Chi Journal of Psychological Research. 2013 Jun 1;18(2):74-81.
11. Goleman DA. Therapists Find Many Achievers Feel They're Fakes. New York Times. 1984 Sep;11:C1.

12. McDowell WC, Grubb III WL, Geho PR. The impact of self-efficacy and perceived organizational support on the imposter phenomenon. American Journal of Management. 2015 Sep 1;15(3):23.

13. Clance PR, Dingman D, Reviere SL, Stober DR. Impostor Phenomenon in an interpersonal/social context: Origins and treatment. Women \& therapy. 1995 Jun 13;16(4):79-96.

14. Cohen MJ, Kay A, Youakim JM, Balaicuis JM. Identity transformation in medical students. The American journal of psychoanalysis. 2009 Mar;69(1):43-52.

15. Mascarenhas VR, D'Souza D, Bicholkar A. Prevalence of Impostor Phenomenon and its association with self-esteem among medical interns in Goa, India. International Journal of Community Medicine and Public Health. 2019 Jan;6(1):355-9.

16. Gottlieb M, Chung A, Battaglioli N, SebokSyer SS, Kalantari A. Impostor syndrome among physicians and physicians in training: a scoping review. Medical education. $2020 \mathrm{Feb}$;54(2):116-24.

17. Higgs, Kathryn Refshauge, Elizabeth Ellis J. Portrait of the physiotherapy profession. Journal of interprofessional care. 2001 Jan 1;15(1):79-89.

18. Smith M, Joy H, Ellis E. Effect of experience on clinical decision making by cardiorespiratory Physiotherapists in acute care settings. Physiotherapy Theory and Practice. 2010 Jan 1;26(2):89-99.

19. Takeuchi R, O'Brien MM, Ormond KB, Brown SD, Maly MR. "Moving forward": success from a physiotherapist's point of view. Physiotherapy Canada. 2008 Apr 15.

20. Topping ME. The Impostor Phenomenon: A study of its construct and incidence in university faculty members..

21. Qureshi MA, Taj J, Latif MZ, Zia S, Rafique M, Chaudhry MA. Imposter syndrome among Pakistani medical students. Annals of King Edward Medical University. 2017 Aug 17;23(2).

22. Clark M, Vardeman K, Barba S. Perceived inadequacy: A study of the imposter phenomenon among college and research librarians. College \& Research Libraries. 2014 May 1;75(3):255-71. 
23. Cowman SE, Ferrari JR. "Am I for real?" Predicting impostor tendencies from selfhandicapping and affective components. Social Behavior and Personality: an international journal. 2002 Jan 1;30(2):11925.

24. Cozzarelli C, Major B. Exploring the validity of the Impostor Phenomenon. Journal of social and clinical psychology. 1990 Dec;9(4):401-17.

25. Kumar S, Jagacinski CM. Imposters have goals too: The imposter phenomenon and its relationship to achievement goal theory. Personality and Individual Differences. 2006 Jan 1;40(1):147-57.

26. Oriel K, Plane MB, Mundt M. Family medicine residents and the Impostor Phenomenon. Family Medicine-Kansas City-. 2004 Apr 1;36(4):248-52.

27. Villwock JA, Sobin LB, Koester LA, Harris TM. Impostor syndrome and burnout among American medical students: a pilot study.
International journal of medical education. 2016;7:364.

28. Paladugu S, Wasser T, Donato A. Impostor syndrome in hospitalists-a cross-sectional study. Journal of community hospital internal medicine perspectives. 2021 Mar 4;11(2):212-5.

29. Regan PA, Shumaker K, Kirby JS. Impostor syndrome in United States dermatology residents. Journal of the American Academy of Dermatology. 2020 Aug 1;83(2):631-3.

30. Henning K, Ey S, Shaw D. Perfectionism, the Impostor Phenomenon and psychological adjustment in medical, dental, nursing and pharmacy students. Medical education. $1998 \mathrm{Sep} ; 32(5): 456-64$.

How to cite this article: Kansara VP, Kumar N, Pabla S. Prevalence of impostor phenomenon in physiotherapy professionals: a pan India survey. Int J Health Sci Res. 2021; 11(10): 322-331. DOI: https://doi.org/10.52403/ijhsr.20211042 